\title{
Terapia cognitivo conductual integrativa
}

\author{
Héctor Fernández-Álvarez y Javier Fernández-Álvarez ${ }^{1,2}$ \\ ${ }^{1}$ Fundación Aiglé, Buenos Aires, Argentina \\ ${ }^{2}$ Universitat Jaime I, Castellón, España
}

Integrative cognitive behavioral therapy

\begin{abstract}
In the last years, interest has grown to integrate the diverse frameworks that coexist within Cognitive Behavioral Therapy (CBT). However, CBT history identified such integration from its beginning. Along with this historic evolution, characterized by a dialectical process of tensions and integration, this article depicts the principles that facilitate an integration in two directions. First, we describe the fundamentals upon which integration of frameworks coexist in the field of CBT. Second, the thesis that CBT can act as a pivot enhancing the integration of theoretical models. Three core ideas serve as potential articulators of the integration movement if adopted. a) To apply a broad information processing framework; b) To design and apply interventions focused both on the behavioral and experiential level; c) The paramount importance of personality.
\end{abstract}

Key words: cognitive behavioral therapy; integration; psychotherapy.

Resumen: En los últimos años ha surgido un interés creciente por integrar los diversos enfoques que cohabitan en la terapia cognitivo conductual (TCC). Sin embargo, la integración en la TCC puede ser identificada desde sus comienzos. Este artículo presenta dicha evolución caracterizada por un proceso dialéctico de tensiones e integración, exponiendo los principios que pueden promover la integración en dos direcciones. Primero, las bases sobre las que es posible asentar la integración de los enfoques que coexisten en el campo de la TCC. Segundo, la tesis de que la TCC puede ser el pivote que potencie la integración de los modelos terapéuticos. Los tres principios nucleares que sirven para facilitar dicha integración son a) El empleo de un modelo amplio de procesamiento de información; b) El diseño y la aplicación de intervenciones que atiendan a los niveles de la conducta y la experiencia; y c) El papel central de la personalidad.

Palabras clave: Terapia cognitiva conductual; integración; psicoterapia.

\section{Introducción}

Hablar de Integración dentro del campo de la Terapia Cognitiva en la actualidad es un hecho habitual, que no estaba presente 10 años atrás. En ese entonces hubiera sido algo tan inhabitual como fue impactante escuchar a David Clark decir, en la sesión de apertura del último Congreso de la WCBCT, en Melbourne, que la terapia cognitiva conductual (TCC) se encuentra en un buen momento para pensar y operar integrando los diferentes enfoques que conviven dentro de su territorio. Como

Recibido: 28 marzo 2017; aceptado: 22 junio 2017

Correspondencia: Héctor Fernández-Álvarez, Fundación Aiglé, Virrey Olaguer y Feliú 2679, Ciudad de Buenos Aires, Argentina, hfa@aigle. org.ar
Clark mencionara en esa oportunidad, en nuestra disciplina se desarrollaron diversos abordajes y muchos modelos a través de olas sucesivas, pero más allá de sus diferencias, los representantes de todos ellos (o casi todos) concurren y se sienten convocados por ese tipo de eventos, señal de que se sienten parte de un espacio común.

Hoy en día, en nuestra disciplina la integración no es algo que está por ocurrir; es algo que ya está ocurriendo. Una muestra cabal de ello es la variedad de enfoques que emplean la denominación de «integrativo», para calificar modelos específicos de tratamiento de TCC. Uno de los primeros ejemplos fue la presentación de Casey, Oei y Newcombe (2004) que apelaba a integrar el rol de las cogniciones positivas y negativas en el modelo de abordaje para el trastorno de pánico. En ese trabajo, los autores sostienen que, para una mejor comprensión del tras- 
torno, resulta útil agregar, al modelo tradicional apoyado en el abordaje de las interpretaciones catastróficas, el rol que juegan los mecanismos de autoeficacia tal como fueran examinados tiempo atrás por Bandura. El modelo de Casey ha ejercido significativa influencia en la construcción teórica. Un buen ejemplo es la presentación de Sandín, Sánchez-Arribas, Chorot y Valiente (2015) que partiendo dicho modelo, propone un enfoque tripartito del pánico integrando, además, la variable de la sensibilidad a la ansiedad. La evaluación de esos tres componentes puede llevarse a cabo mediante instrumentos, como el CATP (Cuestionario Abreviado de Trastorno de Pánico) que ha demostrado excelentes propiedades psicométricas de confiabilidad y validez (Sánchez-Arribas, Chorot, Valiente, y Sandín, 2015).

Otros ejemplos de abordajes integrativos de terapia cognitivo conductual incluyen, cronológicamente, las siguientes propuestas:

Mansell (2007) presentó un enfoque integrativo de abordaje para el trastorno bipolar, apoyado en los hallazgos vinculados con la presencia de fenómenos transdiagnósticos a lo largo del espectro psicopatológico. Varias características del trastorno bipolar, como la elevada comorbilidad de la disfunción con otras perturbaciones, y el peso de los factores ambientales, justifican según dicho autor, expandir la terapia cognitiva en esa dirección. Recientemente el grupo liderado por él en la Universidad de Manchester desarrolló un nuevo enfoque de TCC, denominado TEAMS (Think Effectively About Mood Swings), un modelo cognitivo integrativo cuyo foco es ayudar a los pacientes a incrementar la autoconciencia de evaluaciones extremas de los estados internos, utilizando técnicas metacognitivas junto con las técnicas clásicas conductuales cognitivas. Algunos estudios preliminares han mostrado cierta efectividad y actualmente se están realizando nuevos estudios empíricos para evaluar su eficiencia (Joyce, Tai, Gebbia, y Mansell, 2016).

Nuevos desarrollos caracterizados como enfoques integrativos de terapia cognitiva fueron apareciendo paulatinamente en centros de desarrollo en distintos países. Por ejemplo Khetrapal (2007), en India, ofreció un enfoque centrado en el TDAH, basado en el modelo cognitivo integrativo de las emociones que Power y Dalgleish habían elaborado años antes. Wikowski y Robinson (2010) expusieron un modelo integrativo enfocado en las reacciones de cólera y en las conductas agresivas en el que enfatizan la articulación de tres procesos cognitivos básicos (interpretaciones hostiles, procesos rumiativos y control excesivo), que contribuyen a generar dichas conductas. Este modelo es un ejemplo elocuente del interés que viene despertando la integración en el campo teórico y en la ciencia básica.
En el campo de la clínica, en los últimos años, asistimos a un creciente surgimiento de nuevos modelos de terapia cognitiva integrativa, aplicados a condiciones muy diversas como esquizofrenia (Howes y Murray, 2014), bulimia nerviosa (Wonderlich, Peterson, y Smith, 2015) y, más recientemente, para ansiedad en general (Alladin, 2016). Esta última presentación enfatiza la incorporación de intervenciones de hipnosis terapéutica en el marco de un programa amplio de procedimientos. El abordaje de la depresión, una de las condiciones clínicas más habituales, y que constituye la patología emblemática de la terapia cognitiva, no ha escapado al desarrollo de abordajes enfocados desde una perspectiva cognitiva integrativa. Un ejemplo ya clásico es el estudio de Constantino y colaboradores (2008). En un área asociada, como es el duelo prolongado, los efectos emocionales disfuncionales han sido abordados recientemente también desde esta perspectiva por un grupo de investigación en Alemania (Rosner, Bartl, Pfoh, Kotoucová, y Hagl, 2015).

\section{Objetivos de este artículo}

Es ampliamente reconocido que uno de los hitos fundacionales de la terapia cognitiva está constituido por las aportaciones de Albert Ellis y Aaron Beck. El foco primordial en el que se enfocó Beck en sus inicios fue, como es sabido, el abordaje de la depresión y, por extensión, de las perturbaciones emocionales. Dos notas sobresalientes caracterizaron este nuevo abordaje terapéutico: la importancia otorgada a las variables mediadoras en la generación y el curso de los procesos disfuncionales, y la prevalencia asignada a las operaciones cognitivas (y en particular a ciertos procesos de pensamiento) en la gestación y mantenimiento de las perturbaciones emocionales.

El rápido éxito obtenido por esta propuesta terapéutica, refrendado por el famoso estudio colaborativo sobre depresión, que promovió el Instituto Nacional de Salud Mental de los Estados Unidos (Elkin et al., 1989), que incluyó la terapia cognitiva como una de las intervenciones puestas a prueba, contribuyó decididamente a generar un prestigio importante de este nuevo abordaje terapéutico. Dicho prestigio se tradujo en un fenómeno de reconocimiento social acentuado y favoreció una significativa arborización de sus aplicaciones y también el surgimiento de nuevas propuestas teóricas y técnicas. En pocos años, la terapia cognitiva fundada por Beck, estaba siendo indicada como un tratamiento de elección para una gran diversidad de condiciones clínicas a lo largo de todo el espectro psicopatológico. Esta multiplicación de indicaciones estuvo acompañada con el surgimiento de 
nuevos abordajes terapéuticos, algunos cercanos del modelo clásico y otros que fueron presentados como propuestas alternativas y conceptualmente divergentes al enfoque original.

Ejemplos de cada una de estos dos desarrollos lo constituyen la Terapia Centrada en Esquemas de Jeffrey Young y la Terapia de Aceptación y Compromiso de Steven Hayes. En pocos años el mundo de la terapia cognitiva se había convertido en un campo heterogéneo y diversificado de propuestas terapéuticas, algunas de las cuales postulaban la necesidad de recuperar los principios del análisis y modificación de la conducta. A continuación, se construyó una historiografía que describía el desarrollo de este campo como una sucesiva formación de olas progresivas a través del tiempo.

Nuestro propósito no es presentar un nuevo modelo específico de terapia sino exponer los principios que pueden favorecer el avance hacia una mayor integración de la TCC en dos direcciones. Por un lado, presentar las bases sobre las que es posible asentar la integración de los distintos enfoques que cohabitan en este heterogéneo campo de la terapia cognitvo-comportamental. Al mismo tiempo, defender la tesis de que la terapia cognitiva, así entendida, puede ser el pivote que potencie el movimiento, ya existente dentro del mundo de la psicoterapia, hacia una integración de los distintos modelos terapéuticos. El enfoque cognitivo conductual puede servir como eje integrador de la psicoterapia en general y ayudar a la organización paradigmática de la disciplina.

\section{¿Qué significa integración?}

Hace varias décadas, al tiempo que se multiplicaban los modelos de psicoterapia (y en parte probablemente debido a esa misma proliferación), se abrió un intenso debate respecto a la factibilidad de que pudiera lograrse una integración de la disciplina. Un activo movimiento de académicos y profesionales impulsó múltiples proyectos con el fin de concretar dicho objetivo. La creación, en 1983, de la Sociedad para la Exploración de la Integración de la Psicoterapia es un hito significativo, que fue acompañado en estos más de 30 años por una prolífica producción de la organización (textos, investigaciones, eventos, etc.). De inmediato se levantaron voces que pregonaron tanto el apoyo como el cuestionamiento a esa iniciativa. Entre los argumentos críticos más frecuentes, se destacó la eventual dificultad para articular, de manera coherente, vectores teóricos de diversa procedencia, especialmente enfocados en la aparente irreductibilidad conceptual de los enfoques más abarcadores (psicoterapia psicodinámica, humanística-existencial, sistémica y cognitivo-conductual). De la presunta imposibilidad de articulación epistémica entre distintos modelos se derivaban consecuencias prácticas, en particular las barreras aparentes para la transferencia interteórica de técnicas terapéuticas. Esto último fue desmentido en los hechos. Muchas herramientas se emplean, con grados variables de adaptación, en una gran diversidad de abordajes.

El cuestionamiento al intento de integración en psicoterapia también subrayó la falta de estudios empíricos de evidencia que apoyaran las propuestas integrativas. Aunque efectivamente no hay datos firmes de evidencia con intervenciones basadas en tratamientos integrativos (ver, p.ej. Nathan y Gorman, 2015) la cuestión merece un examen cuidadoso. Es importante tener en cuenta que los estudios empíricos sobre eficiencia terapéutica favorecieron, por obvias razones metodológicas, las intervenciones apoyadas en procedimientos breves y acotados. Esto trajo como consecuencia, por ejemplo, que el modelo de terapia cognitivo conductual se viera beneficiado para aportar una rápida y abundante cantidad de pruebas, mientras que otros enfoques necesitaron más tiempo para comenzar a reunir la evidencia necesaria. De hecho, uno de los fenómenos más interesantes es la relativización de los resultados de la terapia cognitivo-conductual que ha comenzado a informarse en los últimos años (Johnsen y Friborg, 2015)

Por lo tanto, es lógico que debamos esperar la evolución de la investigación de enfoques integrativos, tanto de la teoría como de sus aplicaciones, para que alcancen la madurez suficiente que permita reunir las pruebas de evidencia requeridas. Hoy existen buenas razones para pensar que esa investigación está progresando de manera firme (Castonguay, Eubanks, Goldfried, Muran, y Lutz, 2015; Boswell, 2016). Mientras tanto, la tendencia a la integración parece afirmarse claramente como resultado del desarrollo de la psicoterapia en general, como una respuesta «natural» a los obstáculos y limitaciones que han ido encontrándose en las intervenciones psicoterapéuticas y la identificación de puentes de asociación muy firmes entre enunciados procedentes de distintos modelos teóricos.

Abordando el núcleo duro de los cuestionamientos que se hacen a la posibilidad de construir una psicoterapia cognitiva (y una psicoterapia en general) de carácter integrativo, nos encontramos con factores de mucho peso específico (pero con poca validez conceptual) que merecen ser seriamente interpelados. Mencionamos a continuación, fundamentalmente, dos de estos factores. El primero es la existencia de un agresivo movimiento de resistencia que se opone a la integración como expresión de una fuerte política de defensa de intereses sectoriales. Paris (2013) afirma: 
La historia de la psicoterapia, donde cada abordaje terapéutico incluyó la creación de una secta separada, condujo al marketing de cientos de métodos, muchos de los cuales pueden ser identificados por un acrónimo de tres letras, fácil de recordar. La integración de la psicoterapia debe luchar contra esas tradiciones, así como contra las fuerzas del mercada que las sustentan. (Paris, 2013, p. 100).

Este fenómeno tiene alcances muy potentes, ligados con las tramas políticas que condicionan el desarrollo de la psicoterapia, como ocurre con cualquier disciplina académica y/o profesional. La construcción del conocimiento científico está siempre expuesto a esos avatares y quienes trabajan en esa labor deben ser pacientes y estar dispuestos a sortear esas dificultades.

La otra razón será el eje medular de este artículo y se vincula con la definición y el alcance que tiene una teoría de la integración de la psicoterapia como condición necesaria para alcanzar propuestas terapéuticas más consistentes con la realidad que rige en la práctica clínica. ¿Qué queremos decir cuando hablamos de psicoterapia y de TCC integrativas?. Para comenzar, es indispensable repasar el concepto de integración pues con cierta frecuencia, tanto la defensa como la crítica de la integración en la psicoterapia, adolecen de cierta ligereza conceptual.

¿Qué significa integrar en la experiencia humana en general? Integrar se refiere a la acción mediante la cual se reúne un conjunto de elementos que pasan a formar parte de una totalidad y que, de no mediar esa acción, permanecen disgregados entre sí. Por lo tanto esto supone varias premisas, empezando por el hecho de que sólo es posible integrar componentes que tienen una necesaria fidelidad de conjunto. La integración supone, de este modo, una acción factible a partir de elementos disponibles cuya concreción producirá un efecto superador en algún sentido (por ejemplo en sus efectos, sus resultados, su funcionalidad, etc.).

La raíz del término integración y sus múltiples derivaciones tiene significados convergentes que siempre remiten a dicho carácter superador. Se manifiesta en una gran multiplicidad de campos gnoseológicos y está presente en una elevada diversidad de territorios. En psicología, por ejemplo, encontramos esa expresión cuando se habla de integración de conocimientos o integración de recuerdos. En ambos casos, se describen acciones mediante las cuales la mente es capaz de reunir fuentes diversas de elementos o inputs en torno a una síntesis operativa. Integrar conocimientos no es sumar elementos dispuestos en forma serial sino articular un conjunto diverso de fuentes de información que permiten descu- brir una realidad latente o inobservada previamente. Los docentes, especialmente en el curso de la enseñanza primaria, prestan gran atención a la competencia de los niños respecto a su capacidad para integrar o no los conocimientos. En la misma línea hablamos de integración de recuerdos para describir esa sorprendente operación de la memoria en que la mente convierte una sucesión de imágenes, incorporadas en momentos separados, en una película, reuniendo fragmentos depositados a través del tiempo en una secuencia histórica.

Si uno quiere observar la amplitud de campos que cubre el concepto de integración puede echar un vistazo a disciplinas tan alejadas como las matemáticas, la computación y la sociología. En el campo de las matemáticas la integral es una función descubierta siglos atrás que permite sumar elementos infinitesimales sencillos para reducir la complejidad del área a calcular, en computación se habla de circuitos integrados en referencia con estructuras comprimidas que permiten multiplicar competencias y funciones mejorando costos y rendimientos y en sociología se utiliza el concepto de integración social para definir los fenómenos que atañen a la capacidad de un grupo social para favorecer la comunicación entre las personas y el sentido de pertenencia de los seres humanos. Concepto éste relevado por las Naciones Unidas en su campaña sobre defensa de los derechos humanos. Actualmente vivimos muchas circunstancias en las que muchos eventos a lo ancho del planeta ponen a prueba la capacidad de la sociedad contemporánea para favorecer o no la integración social (valgan como ejemplo las crisis migratorias).

Una conclusión que puede extraerse de esta diversidad de acepciones del concepto de integración es que dicho término no es sinónimo de unificación. Este último supone una operación de otro orden respeto de las partes. La integración de un campo no implica la eliminación de los componentes o las partes, sino la articulación de las mismas. La propuesta de apelar a un programa para integrar los distintos modelos y enfoques que forman el campo de la psicoterapia no responde solamente a la tendencia natural a la integración que se observa en cualquier ámbito de la ciencia. En el caso de nuestra disciplina, aparece como la opción más favorable para abordar los fenómenos clínicos en virtud de su extraordinaria complejidad. Al mismo tiempo, la búsqueda de la integración debe entenderse como un programa abierto de investigación. En ese sentido, es de esperar que la evolución de la psicoterapia y las progresivas novedades teórico-técnicas que vayan emergiendo en su desarrollo requieran propuestas de integración progresivas que den cuenta de esa creciente complejidad. Un programa para integrar la psicoterapia no debe 
entenderse como una tarea con fines conclusivos sino como una persistente manera de responder a los nuevos interrogantes que suscita la disciplina.

\section{Tensiones y acercamientos de la TCC a una concepción integrativa}

La factibilidad de crear un marco integrativo para la TC en el momento actual tiene fuerte sustento en la evolución que experimentó nuestra disciplina a lo largo de su historia. Nos proponemos, a continuación, mostrar que ha habido una estrecha relación entre la TC y el movimiento integrativo, una relación dual en la que, a través del tiempo, se pueden observar tensiones sucesivas traducidas en posturas y actitudes que alternaron entre la confrontación y el acercamiento entre ambos. La orientación de ese proceso pone en evidencia la tendencia hacia un acercamiento progresivo y esa es la tesis que apoyamos: que la TCC avanza hacia un modelo integrativo más pleno. Presentamos, a continuación, una síntesis de los acontecimientos más relevantes que marcan esa tendencia.

\section{Los antecedentes}

Como sabemos, la TC surgió en la década de 1960. Su sistema conceptual se apoyó sobre dos pilares: a) la importancia de las variables mediadores entre estímulos y respuestas y b) la prevalencia de las operaciones cognitivas en el curso de las disfunciones emocionales. El primer pilar coincidía con el enfoque conductista de terapia, pues puso el foco de las intervenciones en el aquí y ahora y acentuó los cambios en los síntomas y en la conducta, pero difería en el papel de las representaciones y en el rol de los estados mentales. En esto se acercó a la naciente ciencia cognitiva, surgida en los laboratorios del MIT, aunque nunca fue un modelo ajustado estrictamente a los principios de dicha ciencia. El segundo pilar, escasamente reconocido, mostró la influencia de las investigaciones de la psicología del yo, llevadas a cabo por Rapaport y sus colegas en el Austin Riggs Center sobre los procesos de pensamiento en el marco de la teoría psicoanalítica, donde Beck había recibido entrenamiento entre 1950 y 1952.

La naciente TC tuvo sus orígenes, por ello, en un híbrido teórico, en un momento en que la psicoterapia era duramente cuestionada como hemos señalado anteriormente. $\mathrm{Y}$ en un momento en el que también Wolpe acababa de formular, con mucha fuerza, el principio de inhibición recíproca asociado a la desensibilización sistemática, un aporte conductista que resultaría fundamental para el desarrollo de las técnicas de exposición, una de las téc- nicas más utilizadas en toda la psicoterapia y en la TC en particular.

Lo que más nos interesa aquí es destacar que, desde sus inicios, la TC se ofreció como un método de tratamiento específico y más eficiente para enfrentar la patología más prevalente de su tiempo: la depresión. El modelo original se basó en un postulado fundamental: la existencia de tres niveles cognitivos que filtran los datos de nuestra experiencia: los pensamientos automáticos, las creencias o supuestos intermedios y los esquemas nucleares. El modelo sostenía que dichos niveles están estructurados en un orden jerárquico, en correspondencia con la teoría de los estados mentales de la ciencia cognitiva. Estos supuestos han generado debates en el campo epistemológico.

Por ejemplo, Leder (2016) sostiene que:

El modelo de TCC se apoya en el supuesto de que nuestras cogniciones tienen una estructura jerárquica que es coherente, lógica y accesible por la conciencia. El contenido de todas las cogniciones están compuestas de más contenido cognitivo general. Si los pacientes pueden identificar introspectivamente sus pensamientos automáticos, el modelo cognitivo predice que podrán identificar los esquemas más generales que subyacen a esos pensamientos. Sin embargo, existen serios problemas con la sustentabilidad del modelo jerárquico y sus supuestos sobre la precisión de las cogniciones que son identificadas y desafiadas en terapia (p. 6).

Sus aplicaciones fueron, más allá de esos debates, muy exitosas y alentaron su extensión a otras condiciones clínicas. En primer lugar, al tratamiento de la ansiedad y, luego, a las formas más severas y complejas de la psicopatología como los trastornos de personalidad (TP) y la esquizofrenia. En pocos años, la TC se convirtió en un método terapéutico útil para (casi) todo el espectro psicopatológico

\section{Primera tensión}

El surgimiento de la TCC en la temprana obra de Beck, significó una ruptura fundamental en el panorama de la psicoterapia. Entre las muchas facetas que asumió esa ruptura, una de las más importantes, fue el apoyo de Beck a la idea de que las intervenciones terapéuticas específicas son los principales responsables de la curación del paciente. Las técnicas o los procedimientos instrumentales cognitivos fueron presentados como los promotores del cambio y la eficiencia terapéutica (lo que años después sería ásperamente debatido). Por la misma época (en 1961), Jerome Frank publicó un libro donde 
hizo una presentación que conmovió el campo de la psicoterapia y habría de alcanzar un prestigio sostenido: la tesis de los Factores Comunes, que hoy podemos observar como la formulación primigenia de un modelo de integración. Otras formulaciones del abordaje integrativo como el eclecticismo, la integración teórica y la asimilación integrativa aparecerían años después.

La tesis de los factores comunes sentó la base de que toda psicoterapia necesita contar con una serie de condiciones necesarias (como la empatía, un ámbito de seguridad y confianza y la escucha atenta y receptiva) para poder ser eficiente. La tesis de Frank sostenía que estos factores eran comunes a todos los modelos terapéuticos y no dependían del tipo específico de técnicas que se utilizaran en cada caso. En este sentido, la nueva TC de Beck se situaba en el polo opuesto, pues sostenía con firmeza la idea de que los factores específicos como la exposición y la restructuración cognitiva eran, por el contrario, los principales responsables del cambio terapéutico.

Más de 50 años después de aquella presentación de Frank, el papel de los Factores Comunes y la confrontación con el peso que tienen los Factores Específicos (o técnicos) sigue siendo una fuente de intensos debates. Muchos autores, como Wampold, enfatizan la importancia de los primeros y consideran que la Alianza Terapéutica es la variable que tiene mayor influencia sobre los resultados terapéuticos (Wampold, 2015). Otros, como Barlow, enfatizan el peso de los factores y las guías de tratamiento como el factor de mayor relevancia. Sólo recientemente ambas posiciones parecen haber iniciado un acercamiento (Hofmann y Barlow, 2014; Laska, Gurman, y Wampold, 2014)

\section{Integración: Primer movimiento a favor}

Los logros iniciales que obtuvo la TC no sólo extendieron sus aplicaciones a nuevas condiciones clínicas. Junto a ello impulsaron la aparición de enfoques alternativos, algunos de los cuales surgieron como variantes dentro de la corriente clásica mientras que otros tomaron rumbos teóricos más divergentes. Uno de éstos, muy pujante, fue la Terapia Dialéctico Conductual (TDC) de Linehan, presentada con un marcado carácter integratitvo, como se puede observar en este enunciado (Heard y Linehan, 1994):

La TDC es integrativa, en el sentido dialéctico y del desarrollo de esa palabra, con el significado de que enfatiza el proceso dialógico abierto en el que las diferencias son examinadas y una nueva integración es bienvenida... La TDC es la primera psicoterapia para el trastorno de personalidad borderline y una los pocas psicoterapias in- tegrativas con sostén empírico publicado, basado en un diseño experimental aleatorizado. (Heard y Linehan, 1994, p. 56).

La fuerza del incipiente movimiento de integración en psicoterapia y la eficiencia alcanzada por la TC promovieron, en ese período, la gestación de programas eclécticos y de integración teórica, algunos de los cuales alcanzarían una importante difusión, como en el caso de la Terapia Cognitivo-Analítica, desarrollado por Ryle, que se diseminó en el Sistema nacional de salud de Gran Bretaña.

\section{Segunda tensión}

En los años siguientes la tensión se acentuó, especialmente a partir de un cisma que ocurrió al interior de la joven TC Uno de los ejemplos más elocuentes de ello quedó registrado en el primer número del Journal of Cognitive Therapy, publicado en 1987 . Su contenido estuvo limitado a dos artículos, uno de Aaron Beck y otro de Michael Mahoney. Representaban posturas teóricas muy contrastantes. Como ocurriera con otros abordajes de la psicoterapia, la TC enfrentaba tempranas y profundas disidencias. Beck expuso en ese artículo una síntesis del modelo clásico de TC, al que Mahoney consideraba rígidamente racionalista y al que oponía, a su vez, un enfoque constructivista y posracionalista, uno de cuyos líderes era Vittorio Guidano. Es interesante observar que pocos años antes, Guidano había generado una profunda conmoción en el ámbito cognitivo al presentar un texto (junto con Giovanni Liotti) que postulaba la necesidad de relevar el papel de las emociones en la terapia. Ese aporte anticipó tempranamente, sin dudas, la importancia que tendría el estudio de la emociones y su regulación varios después. La confrontación entre la TC clásica y el enfoque de TC constructivista marcó una señal de franco antagonismo a la idea de la integración.

\section{Integración: Nuevo acercamiento}

Pocos años después (1995), con ocasión de un Congreso Internacional de la especialidad, en Copenhagen, el evento fue presentado por su presidenta con el propósito de favorecer una integración entre ambos enfoques. Más allá de la anécdota, hubo entonces hechos importantes que acentuaron el acercamiento de la TC a una perspectiva integrativa. El primero se vincula con la expansión que experimentó el modelo clásico. Como hemos señalado, la TC original rápidamente extendió sus aplicaciones y pasó a operar como un método terapéuti- 
co destinado a patologías más complejas, en particular los trastornos de personalidad (TP). Para abordar esos trastornos, el modelo original necesitó no solamente ampliar el foco de sus intervenciones sino incorporar nuevas estrategias y procedimientos. Esto quedó claramente reflejado en el texto respectivo que presentaron Beck y sus colegas en 1990. De manera más elocuente aún, cambios conceptuales profundos quedaron plasmados en el desarrollo de la Terapia de Esquemas a la que Young, Klosko y Weishaar (2003) definieron como:

Un modelo amplio, integrativo. Como tal, se superpone de manera considerable con otros sistemas de psicoterapia, incluyendo los modelos psicodinámicos. Sin embargo, la mayoría de estos abordajes son más estrechos que la terapia de esquemas, tanto en términos del modelo conceptual como en relación con las estrategias terapéuticas. (Young, 2003, p. 61).

Las relaciones entre TC e integración se hicieron más fluidas y, al mismo, más complejas. Un texto de Alford y Beck (1997), recogió claramente esa dualidad. Expusieron allí potentes argumentos que sostenía el poder de la TC para favorecer la integración de la psicoterapia. El texto comenzaba haciendo una crítica dura a la ideología «oficial» del movimiento de integración considerando que carecía de bases científicas, por manejarse con definiciones imprecisas y por emplear de manera ambigua el principio de los factores comunes. Proponía adoptar el modelo de la TC como una base científica sólida, capaz de facilitar «un lenguaje común para la práctica clínica y como un abordaje técnicamente ecléctico que puede ganar coherencia gracias a la teoría cognitiva».

El primer movimiento concreto en esa dirección fue la integración de la TC con el enfoque conductista. En realidad, algunas técnicas comportamentales y la exposición en particular, fueron identificadas rápidamente como un procedimiento nuclear que la TC utilizaba en sus aplicaciones. Este hecho condujo a una convergencia «natural» entre conductismo y cognitivismo, dando lugar a que la TC formara parte, de allí en más, de un modelo más amplio: la TCC como hoy se conoce. Como contrapartida, esto marcó el distanciamiento de los representantes de la TC posracionalista, enrolados en una perspectiva constructivista radical.

\section{Integración: Tercera tensión}

En ese momento, tomaron forma nuevos desarrollos teórico-técnicos que propusieron recuperar la relevancia de la terapia comportamental, opacada durante algunos años por la ofensiva de la TC. Esos desarrollos, junto con la presentación de nuevos modelos terapéuticos como la terapia de aceptación y compromiso (TAC) y la TDC fueron acompañados de fuertes críticas a la TC clásica, a la que adjudicaban la dependencia de un marco epistemológico realista, el empleo de intervenciones enfocadas en los contenidos, en desmedro de las funciones, y el centrar los objetivos terapéuticos atados a un modelo supresor de síntomas. Varios autores, liderados por S. Hayes, propusieron un nuevo enfoque centrado en el rescate del funcionalismo contextual, en una firme validación de la experiencia disfuncional y en otorgar prevalencia a una actitud de aceptación y compasión en lugar de promover la inducción al cambio. Se elaboró una historiografía del movimiento de la TCC en tres olas sucesivas, integradas en una evolución progresiva de la terapia.

La TAC no sólo se presentó como un enfoque alternativo teórica y técnicamente sino que generó un movimiento institucional de gran despliegue, generando durante algunos años una fuerte competencia con la TC tradicional. El énfasis que puso en el aspecto experiencial del cambio y el impulso que le dio a nuevas formas de intervención, como el empleo de entrenamiento en técnicas de meditación, instalaron una tensión muy intensa durante algunos años en el seno de la disciplina.

El empleo de técnicas de meditación orientales (y del budismo en particular) significó un aporte relevante pero, al mismo tiempo, merece un examen crítico. Hay en ello otro ejemplo del efecto de halo consecutivo al éxito ya mencionado. Este recurso, utilizado en principio para la asistencia de pacientes con enfermedades crónicas y terminales, se extendió luego (con cierta desmesura) a una enorme diversidad de situaciones clínicas. Se han reunido pruebas empíricas de su eficiencia, pero es mucho lo que todavía resta investigar para conocer cuál es el alcance de sus aplicaciones en general. Sobre todo, no es seguro que resulte superior a otras formas de meditación.

En los fundamentos que justificaron su empleo se cometieron algunas falacias teóricas y conceptuales. En primer lugar, se contrastaron las prácticas de meditación orientales con el cuerpo teórico de la ciencia occidental. El paradigma de la ciencia en occidente merece ser (y ha sido) criticado para discutir sus alcances y limitaciones en el terreno de la clínica y la psicoterapia. Pero, en ese marco, debe confrontarse con otras formas de saber empírico, propias de diversos marcos culturales, incluido el oriental. Las prácticas de meditación budista pueden ser comparadas, en cambio, con otras formas de meditación, como las que se difundieron en la cultura occidental. Por ejemplo la meditación teresiana, cisterciense o ignaciana en la tradición cristiana y también con prácticas de la tradición judía e islámica (cultura sufí). 


\section{Otra vuelta hacia la integración}

Como señalamos, una de las notas sobresalientes de este nuevo movimiento ha sido la incorporación de técnicas de meditación a la terapia, sobresaliendo el empleo de prácticas de mindfulness, un nuevo recurso que alcanzó una explosiva expansión en poco tiempo. La TCC dio pruebas, al incorporar esas prácticas como parte de su arsenal terapéutico, de una doble ductilidad para favorecer la integración. Por un lado, sumando a las clásicas estrategias de cambio centradas en la exposición y la reestructuración, estos nuevos recursos enfocados en el ejercicio de la atención plena. Por otro lado, integrando principios de la tradición occidental y oriental.

Por otra parte, la brecha entre las diferentes olas de TC comenzó a cerrarse al encontrarse puentes entre los distintos enfoques y principios para sumar diferentes intervenciones. Una de las razones que habían sostenido los abordajes de Tercera Ola, había sido el cuestionamiento de que los beneficios que brindaba la TC clásica no respondían a los supuestos invocados por ese modelo sino, fundamentalmente, al empleo de recursos comportamentales y no cognitivos. Además, los defensores de la Tercera Ola sostuvieron que su enfoque teórico era radicalmente diferente al de la TCclásica. Esto puede extenderse al contraste entre los abordajes metacognitivos y la TCclásica (Garay y Keegan, 2016). Sin embargo, tales diferencias no parecen tan radicales. Por ejemplo, todos ponen el foco terapéutico en trabajar con cogniciones accesibles por vía conciente y en modificar cogniciones negativas, aunque a través de diferentes estrategias. De allí se desprende que, en la medida que sea posible identificar los puntos en común entre las terapias de segunda y tercera generación será factible desarrollar estrategias terapéuticas integrativas utilizando principios extraídos de ambos enfoques. Eso es, precisamente, lo que proponen Hallis, Cameli, Dionne y Knaüper (2015) con un nuevo programa manualizado para el tratamiento grupal de la depresión basado en una combinación de TC y TAC:

Proponemos que al ofrecer una terapia que incluye estrategias tanto de TC como de T.A.C se brinda a los clientes más opciones para tratar los pensamientos y emociones que les causan malestar y, por lo tanto, incrementar la efectividad de la terapia. Según nuestro conocimiento, este es el primer paquete integrativo que combina TC y TAC para el tratamiento de la depresión. (Hallis et al., 2015, p. 187).

En la misma dirección y como expresión de un modelo teórico más abarcador y complejo tenemos el ejemplo de la Terapia de Método de Niveles (TML) basado en la teoría del control perceptual (Carey, Mansell, y Tai, 2015). Desde una perspectiva declaradamente integrativa, este abordaje afirma reconocer y valorar las contribuciones de la primera, la segunda y la tercera ola de TCC, pero no se alinea con ninguna onda específica en particular.

Estas circunstancias son sólo la punta del iceberg de un fenómeno potente y extendido que muestra una pujante actividad en muchos frentes que van marcando hitos progresivos de la integración. Para ilustrar las notas más relevantes de ese fenómeno es necesario detenernos un instante a examinar cuál es la situación de la TCC en la actualidad.

\section{La situación actual}

La TCC adquirió un marcado prestigio y reconocimiento debido a la su capacidad para mostrar sus beneficios con estudios empíricos de eficacia y efectividad. Existen muchas revisiones y metanálisis cuyos resultados aportaron importantes hallazgos (Hofmann, Asnaani, Vonk, Sawyer y Fang, 2012). Las conclusiones más importantes que pueden extraerse de esos estudios son las siguientes:

i) La TCC muestra probada eficiencia al compararla con listas de espera y con tratamientos inespecíficos. También presenta superioridad respecto de otros modelos de psicoterapia para situaciones clínicas específicas, pero esto no apoya la superioridad absoluta del modelo sobre otros enfoques de psicoterapia. ii) El mejor rendimiento se observa en el tratamiento de las perturbaciones emocionales (depresión y ansiedad), pero también hay buenos resultados con otras patologías. iii) La mejoría de los pacientes se evidencia, en especial, en el alivio sintomático, aunque también se verifican mejorías en otras variables del funcionamiento vital. iv) $\mathrm{La}$ TCC contribuye con la mayoría de estudios vigentes en la literatura que prueban la eficiencia de la psicoterapia. A su vez, la mayoría de esos datos corresponden a estudios de eficacia, obtenidos en base a ensayos controlados aleatorizados (ECA).

Esa importante masa de datos dan testimonio de la superioridad operativa y modelística que tiene la TCC Sin embargo, también subsisten varias cuestiones pendientes que muestran aspectos débiles del modelo. Identificamos cuatro aspectos muy importantes:

i) Muchos datos pertenecen a estudios muy recientes y deben ser considerados con cautela. Necesitamos un tiempo bastante prolongado para asegurar la superioridad de un nuevo procedimiento. En investigación, esto 
es un deber. Por ejemplo: una reciente revisión sobre intervenciones en Modificación de Sesgos Atencionales, llegó a la conclusión que, contrariamente a lo que surgía de datos preliminares, dicha intervención es menos eficiente de lo esperado y de las expectativas que había sobre su utilidad clínica (Cristea, Kok, y Cuijpers, 2015). Todavía tenemos importantes cuestiones metodológicas a resolver en el campo de la psicoterapia. ii) Además, las pruebas empíricas que muestran los beneficios de la TC (no solamente en lo que se refiere al alivio sintomático) no garantizan que los fundamentos de sus intervenciones estén sólidamente establecidos. Algunas de las objeciones epistemológicas más importantes a la TCC se enfocan sobre la naturaleza representacional de las creencias y los pensamientos:

Las teorías representacionales de la cognición consideran a las creencias y los pensamientos como representaciones mentales causalmente eficaces de hechos, estados de situación o proposiciones. Los críticos del representacionalismo han sostenido que la TCC confunde los informes individuales del pensamiento (que están representados por su contenido icónico o lingüístico) con los propios pensamientos (que no necesitan tener ningún contenido representacional. (McEachrane, 2009 citado en Leder, 2016, p. 397).

Por otra parte, más allá de sus éxitos, un porcentaje importante de pacientes muestra un rendimiento menos exitoso o fracasa en su tratamiento debido a:

(1) abandonos tempranos

(2) poca estabilidad de los resultados, recaídas y recurrencias sintomáticas

(3) pobre respuesta al tratamiento, especialmente cuando existe una asociación con una disfunción compleja (sobresalen los TP)

Finalmente, una observación importante: en los últimos años se han venido presentando informes sobre estudios empíricos de eficiencia obtenidos con otros modelos terapéuticos. Eso se puede comprobar en diversas fuentes, por ejemplo en los datos de la Cochrane Collaboration. Sobresale el abordaje psicodinámico (Leichsenring et al., 2015) y sus aplicaciones más destacadas se encuentran, también, en el terreno de las perturbaciones emocionales y, más recientemente, en el campo de los TP Los otros enfoques fundamentales de psicoterapia (el sistémico y el humanístico) han comenzado a ofrecer también aportes en ese sentido (Angus, Watson, Elliott, Schneider, y Timulak, 2015; Heatherington, Friedlander, Diamond, Escudero, y Pinsof, 2015).
Un ejemplo notable de esto último se refiere a los resultados obtenidos con los programas de tratamiento on-line. El uso de Internet y los programas asistidos por computadora fueron, en sus inicios, programas basados exclusivamente en el modelo de la TCC (un ejemplo célebre es Beating the Blues). Los primeros estudios metanalíticos en este campo dieron un fuerte apoyo al modelo teórico de la TCC Pero rápidamente, aparecieron resultados favorables sobre estudios empíricos basados en el modelo psicodinámico (Johansson, Frederick, y Andersson, 2013).

Estos hallazgos han dado lugar a un interés creciente de muchos investigadores por trazar más puentes entre diferentes modelos. Entre los grupos de trabajo comprometidos con este objetivo encontramos programas eclécticos de intervención y diversos modelos teóricos. Un hecho importante es que, en general, alguna modalidad o algunos principios de la TCC constituyen un componente nuclear de dichas propuestas. Seleccionamos tres ejemplos:

1. Elevado grado de compatibilidad teórica e instrumental entre el abordaje de la Terapia Dialéctico Conductual y el modelo de Mentalización de Bateman y Fonagy. Se observaron importantes semejanzas en la lógica teórica de ambos enfoques, en consonancia con los hallazgos de que ambos tienen buenas pruebas empíricas de eficiencia terapéutica en el tratamiento del trastorno de personalidad borderline (Swenson y Choi-Kain, 2013).

2. Hayes sostuvo que la TAC tiene conexiones importantes con los principios generales de la psicoterapia humanístico-existencial (Hayes, 2012).

3. Se han llevado a cabo desarrollos teóricos y estudios de metasíntesis para explorar la conexión entre aspectos conceptuales del psicoanálisis y la terapia comportamental: por ejemplo, examinar la traducción interteórica del concepto de transferencia o interpretar la regulación emocional en el psicoanálisis desde la perspectiva del modelo de desensibilización sistemática de Wolpe (Rabinovich, 2016).

\section{Principios organizadores para una terapia cognitiva integrativa}

Existe amplio consenso de que el territorio de la psicoterapia está cubierto por cuatro grandes vectores teóricos: el psicodinámico, el humanista, el sistémico y el comportamental (Längle y Kriz, 2012). La TC apareció como una alternativa teórica diferente, asociada borrosamente con la ciencia cognitiva en ascenso. Sin embargo, 
en el curso de su desarrollo la TC se incluyó en el tronco de la terapia comportamental, formando un eje común, dentro del cual se alojaron diversos enfoques con matices, en algunos casos, muy contrastantes. Creemos que es posible considerar el amplio cauce cognitivo-comportamental como una corriente con un potencial asimilador para operar como eje articulador de los diferentes enfoques que integran ese campo y de las otras perspectivas teóricas. Para eso es necesario desarrollar un modelo que no intente accionar de manera reduccionista respecto de los diferentes abordajes sino apoyarse en ellos y avanzar hacia una elaboración teórica que tenga como eje ciertos principios organizadores fundamentales.

Los tres principios fundamentales que sirven para facilitar dicha integración son: a) el empleo de un modelo de procesamiento de información conectivo amplio; b) la evaluación de los procesos disfuncionales y la elaboración de los diseños terapéuticos apoyadas en examinar de manera conjunta la conducta y la experiencia; c) la consideración de la personalidad y sus disfunciones como eje articulador del diagnóstico y la terapéutica.

\section{Empleo de un modelo amplio de procesamiento de información}

Los seres humanos están sometidos a una incesante tarea constructiva con miras a organizar la siempre dinámica realidad en que les toca vivir y a las cambiantes circunstancias con que se enfrentan a lo largo de su evolución vital. La forma, la consistencia y, por sobre todas las cosas, el nivel de satisfacción alcanzado en ese proceso tiene efectos que se distribuyen en un continuo de funcionalidad / disfuncionalidad. Es funcional cuando el individuo alcanza un balance satisfactorio respecto de su estado general o, en otros términos, cuando logra ejercer un sentido de agencia personal acorde con las metas de su guión personal. A veces, la organización no resulta adecuada y se traduce en estados disfuncionales que afectan el estado físico, psíquico y social del individuo. Las tareas necesarias para organizar la realidad a lo largo del ciclo vital (y para auto-organizarse) es crecientemente compleja y deben ajustarse en el marco de las relaciones dialécticas que tienen lugar entre el individuo y el contexto primario y secundario. Dichas operaciones implican procesar la información que circula en el sistema, que tiene múltiples niveles y una serie de propiedades: recursividad, retroalimentación, equifinalidad, teleología.

Cada ser humano opera como un sistema procesador de significados cuyas complejas y crecientes exigencias no puede ser abarcado con un modelo explicativo apoyado en un modelo de procesamiento lineal o secuencial. Este principio puede aplicarse para dar cuenta de todas las circunstancias que debe enfrentar el individuo: resolver problemas o encontrar soluciones para eventos de la vida cotidiana, manejar sus emociones ante situaciones de carga variable, distribuir sus capacidades para hacer frente a situaciones dilemáticas, etc. Para evaluar y comprender mejor los fenómenos clínicos (y subclínicos) se necesita contar con un dispositivo analítico que pueda abarcar la complejidad del sistema procesal humano que opera con muchas funciones en paralelo. Esta conectividad de la mente y del sistema nervioso que la sostiene, puede abordarse de manera consistente contando con un marco explicativo que tenga en cuenta esa red funcional.

Para operar en ese nivel de análisis necesitamos modelos de procesamiento cognitivo que permitan abarcar esa complejidad: un modelo basado en la teoría de los sistemas dinámicos que pueda dar cuenta de los procesos de organización en tiempo real; un modelo que considere la cognición situada y encarnada que permita explicar los procesos psicológicos en condiciones específicas, como expresión de una determinada configuración física y corporal; un modelo de procesamiento que atienda al carácter extendido de la mente como un sistema con propiedades emergentes que opera en contextos concretos, activando mecanismos de regulación cognitiva, emocional y social (Osbeck, 2009). Un modelo integrativo de análisis de procesamiento, capaz de articular esos niveles, permite contar con un esquema amplio y dinámico con el que es posible traducir los principios conceptuales y operativos de los diferentes modelos de psicoterapia en un lenguaje convergente.

Diseñar y aplicar intervenciones que atiendan a
los niveles de la conducta y la experiencia

En el transcurso de su vida, los seres humanos luchan por encontrar el mejor equilibrio posible entre dos necesidades contrapuestas. Por un lado, la de tener que hacer frente a los cambios que provienen de dos frentes: uno interno, asociado por sus procesos de desarrollo y atado a los factores evolutivos, otro vinculado con los cambios originados en su entorno y que derivan de eventos circunstanciales. Por otro lado, los individuos necesitan preservar la unidad de su sistema personal, de modo de afirmar su identidad y el reconocimiento para sí mismos y frente a los demás. Cambio e identidad son requerimientos sometidos a una tensión permanente.

En su tarea por cumplir con esas necesidades las personas actúan tratando de alcanzar el equilibrio dinámico más satisfactorio posible. La conducta es el conjunto de acciones que el ser humano acomete, de manera incesante, para cumplir con las numerosas exigencias que le de- 
manda la realidad en que vive. Sus efectos o consecuencias pueden ser funcionales o disfuncionales según el grado en que lograr alcanzar un adecuado balance de satisfacción. Esto dependerá de una compleja red de variables, pero siempre estará intencionalmente orientado a lograr la mayor adaptación posible a las circunstancias que le toca vivir.

Para realizar un buen diagnóstico, tanto de una situación funcional como disfuncional, nuestro punto de partida debe ser la exploración y evaluación de los comportamientos implicados en esa situación. En los procesos disfuncionales en general y en los trastornos en particular, los síntomas son las primera expresión que aqueja al paciente. La desregulación emocional, el episodio maníaco o la crisis de pánico, por ejemplo, son fenómenos que podemos comprender y abordar partiendo del examen de las conductas involucradas en esas situaciones. Nuestro primer acceso para el diagnóstico clínico y para pensar una eventual intervención terapéutica es examinar las conductas y ponderar el grado de desadaptación que implican.

Para acceder a ello, hay que tener presente que la actividad psíquica (tanto funcional como disfuncional) no se reduce al incesante despliegue de comportamientos que lleva a cabo cada individuo día a día. Todas las acciones, tanto abiertas como encubiertas, están articuladas y sirven a un sistema de organización que está constituido por la experiencia personal de ese individuo. Ese sistema responde a varios principios: unidad, totalidad, continuidad e intencionalidad y de su constancia depende la identidad, fundamental para asegurar la integridad de la persona. Esto debe ser especialmente tenido en cuenta ante las situaciones disfuncionales, pues también allí el individuo busca encontrar alguna forma de equilibración, aún para situaciones que pueden ser en extremo difíciles de enfrentar. De allí, la imperiosa necesidad de proceder en todo momento la validación de la experiencia disfuncional.

Un buen diagnóstico clínico y una buena intervención terapéutica serán, por lo tanto, aquellos que pueden tener en cuenta, de manera fuertemente integrativa, la perspectiva de las conductas en que se despliega la disfuncionalidad del paciente y la experiencia en la que están insertas esos comportamientos.

\section{El papel central de la personalidad}

La TCC en todas sus variantes y la psicoterapia en general están destinadas a ayudar a personas que presentan situaciones disfuncionales que les provocan alguna forma de dolor o insatisfacción. Muchas veces esto se expresa a través de síntomas. El uso de manuales y guías de tratamiento es de gran utilidad para orientar los abordajes generales en el campo de la salud mental. Pero, las manifestaciones disfuncionales (síntomas, problemas de comunicación, dificultades en la performance, etc.) están siempre ancladas en patrones básicos de la organización personal. En cada individuo, la estructura de su personalidad no sólo tiene poder causal sobre sus acciones sino que modula su experiencia. Estudiar en todos los pacientes la organización de su personalidad es un elemento integrador fundamental para disponer de una evaluación totalizadora de los fenómenos clínicos y para poder construir diseños de intervención terapéuticos que atiendan a la complejidad de la experiencia.

En los últimos años comprobamos que la personalidad incide de manera significativa en todas las patologías y es un componente que debe ser considerado para el diseño terapéutico. Esto no debe aplicarse solamente cuando se detecte un trastorno de personalidad franco y definido. Muchas formas disfuncionales de la personalidad constituyen también dimensiones que inciden en el curso de las perturbaciones del comportamiento y tienen mucha influencia sobre el pronóstico terapéutico. Las perturbaciones emocionales (la ansiedad y la depresión) fueron las primeras situaciones clínicas en las que se comprobó dicha influencia. Pero, en la actualidad, ese fenómeno se ha comprobado como una variable de mucho peso en todo el arco psicopatológico. Los estilos de personalidad (funcionales o disfuncionales) son decisivos para ajustar el abordaje de patologías tan diversas como la depresión mayor, el trastorno de pánico, la ansiedad generalizada o el trastorno bipolar.

\section{A modo de cierre}

En la última década se produjo un giro de enormes consecuencias en nuestra disciplina: la aparición de los abordajes transdiagnósticos. Tiene varias facetas. En primer lugar, la identificación de procesos de psicológicos básicos que operan como funciones activas subyacentes a trastornos mentales variados y heterogéneos. El descubrimiento de este fenómeno permitió dar un paso muy importante hacia una mejor conexión entre la psicoterapia y los procesos básicos. Cada día la investigación contribuye a identificar nuevos fenómenos de esta naturaleza. La regulación emocional es uno de los más estudios en los últimos años.

En segundo lugar, desplazó el foco terapéutico, pasando de intervenciones centradas en trastornos específicos hacia fenómenos psicopatológicos transversales. Se comenzaron a desarrollar y aplicar programas de tratamientos transdiagnósticos, apoyados en principios que 
habían demostrado elevada eficiencia en diversos enfoques terapéuticos. La exposición, la reevaluación cognitiva, identificación y manejo de la evitación experiencial y el incremento de awareness emocional son algunas de los más diseminados. Esto se concretó en un Protocolo Unificado (Barlow et al., 2010), cuyo objetivo final es brindar asistencia masiva y operar en el campo de la atención primaria. Nuevos programas aparecieron rápidamente. Un ejemplo interesante es el que emplea la TML, estructurado en tono a un mecanismo nuclear: la pérdida de control que resulta del conflicto entre metas situadas en diferente nivel de la experiencia y alejadas de la conciencia (Mansell, Carey y Tai, 2013).

El mayor giro que supuso la aparición de los abordajes transdiagnósticos fue instalar la idea de que es posible atravesar condiciones clínicas y herramientas terapéuticas para alcanzar mayor eficiencia con nuestras intervenciones, tratando de ir más allá de lo que los abordajes específicos habían logrado en los años precedentes. Si esto lo extendemos más allá del ámbito circunscripto en que se originaron estos abordajes y pensamos que esa idea puede llevarse a todo el campo de la TCC podremos visualizar con claridad por qué es posible desarrollar una TCCIntegrativa y por qué podemos pensar que eso pueda ayudar más a nuestros pacientes. De manera adicional, ello puede ser el eje articular que ayude a integrar el ancho territorio de la psicoterapia en general.

\section{Conflictos de intereses}

Los autores declaran que no existen conflictos de intereses

\section{Referencias}

Alford, B. A. \& Beck, A. T. (1998). The integrative power of cognitive therapy. New York: Guilford Press.

Alladin, A. (2016). Integrative CBT for anxiety disorders: An evidence-based approach to enhancing cognitive behavioural therapy with mindfulness and hypnotherapy. New York: Wiley.

Angus, L., Watson, J. C., Elliott, R., Schneider, K., \& Timulak, L. (2015). Humanistic psychotherapy research 1990-2015: From methodological innovation to evidence-supported treatment outcomes and beyond. Psychotherapy Research, 25, 330-347. doi:10.1080/10503307.2014.989290

Barlow, D., Farchione, T. J., Fairholme, C. P., Ellard, K. K., Boisseau, C. L., Allen, L. B., \& Ehrenreich-May, J. (2010). Unified Protocol for transdiagnostic treatment of emotional disorders. Oxford: Oxford University Press.

Boswell, J. F. (2017). Psychotherapy Integration: Research, Practice, and Training at the Leading Edge. Journal of Psychotherapy Integration, 27, 225-235. doi: 10.1037/int0000055
Carey, T. A., Mansell, W., \& Tai, S. J. (2015). Principles-based counselling and psychotherapy. New York: Routledge.

Casey, L. M., Oei, T. P., \& Newcombe, P. A., (2004). An integrated cognitive model of panic disorder: the role of positive and negative cognitions. Clinical Psychological Review, 24, 529555.

Castonguay, L. G., Eubanks, C. F., Goldfried, M. R., Muran, J. C., \& Lutz, W. (2015). Research on psychotherapy integration: building on the past, looking to the future. Psychotherapy Research, 25, 365-382. doi: 10.1080/10503307.2015.1014010

Constantino, M. J., Marnell, M. E., Haile, A. J., Kanther-Sista, S. N., Wolman, K., Zappert, L., \& Arnow, B. A. (2008). Integrative cognitive therapy for depression: A randomized pilot comparison. Psychotherapy, 45, 122-134. doi: 10.1037/00333204.45.2.122

Cristea, I. A., Kok, R. N., \& Cuijpers, P. (2015). Efficacy of cognitive bias modification interventions in anxiety and depression. The British Journal of Psychiatry, 206, 7-16. doi: 10.1192/ bjp.bp.114.146761

Elkin, I., Shea, M. T., Watkins, J. T., Imber, S. D., Sotsky, S. M., Collins, J. F., ... \& Fiester, S. J. (1989). National Institute of Mental Health treatment of depression collaborative research program: General effectiveness of treatments. Archives of general psychiatry, 46, 971-982.

Garay, C. J., \& Keegan, E. (2016). Terapia metacognitiva. El síndrome cognitivo atencional y los procesos cognitivos. Revista Argentina de Clínica Psicológica, 25, 125-134.

Hallis, L., Cameli, L., Dionne, F., \& Knáuper, B. (2016). Combining Cognitive Therapy with Acceptance and Commitment Therapy for depression: A manualized group therapy. Journal of Psychotherapy Integration, 62, 186-201.

Hayes, S. C. (2012). Humanistic psychology and contextual behavioral perspectives. Psychotherapy, 49, 455-460. doi: 10.1037/a0027396

Heard, H. L. \& Linehan, M. M. (1994). Dialectical behavior therapy: An integrative approach to the treatment of borderline personality disorder. Journal of Psychotherapy Integration, 4, $55-82$.

Heatherington, L., Friedlander, M. L., Diamond, G. M., Escudero, V., \& Pinsof, W. M. (2015). 25 Years of systemic therapies research: Progress and promise. Psychotherapy Research, 25, 348-364.

Hofmann, S. G., Asnaani, A., Vonk, I. J. J., Sawyer, A. T., \& Fang, A. (2012). The efficacy of cognitive behavioral therapy: a review of meta-analyses. Cognitive Therapy and Research, 36, 427-440. doi: 10.1007/s10608-012-9476-1

Hofmann, S. G. \& Barlow, D. H. (2014). Evidence-based psychological interventions and the common factors approach. The beginnings of a rapprochment. Psychotherapy, 51, 510-513.

Howes, O. D. \& Murray, R. M. (2014). Schizophrenia: an integrated socio-developmental-cognitive model. Lancet, 383, 1677-1687. doi: 10.1016/S0140-6736(13)62036-X

Johansson, R., Frederick, R. J., \& Andersson, G. (2013). Using the internet to provide psychodynamic psychotherapy. Psychodynamic Psychiatry, 41,513-540. doi: 10.1521/pdps.2013.41.4.513.

Johnsen, T. J. \& Friborg, O. (2015). The effects of cognitive behavioral therapy as an antidepressive treatment is falling: A meta-analysis. Psychological Bulletin, 141, 747-68. doi: $10.1037 /$ bul0000015. 
Joyce, E., Tai, S., Gebbia, P., \& Mansell, W. (2016). What are people's experiences of a novel Cognitive Behavioural Therapy for bipolar disorders? A qualitative investigation with participants on the TEAMS trial. Clinical Psychology and Psychotherapy, 24, 712-726. doi: 10.1002/cpp.2040.

Khetrapal, N. (2007). SPAARS Approach: Integrated cognitive model of emotion of attention deficit/hyperactivity disorder. Europe's Journal of Psychology, 3. doi.org/10.5964/ejop. v3i3.404

Längle, A. A. \& Kriz, J. (2012). The renewal of humanism in European psychotherapy: Developments and applications. Psychotherapy, 49, 430-436

Laska, K. M., Gurman, A. S., \& Wampold, B. E. (2014). Expanding the lens of evidence-based practice in psychotherapy: A common factors perspective. Psychotherapy, 51, 467-481. doi: $10.1037 / \mathrm{a} 0034332$

Leder, G. (2016). Know thyself? Questioning the theoretical foundations of cognitive behavioral therapy. Review of Philosophy and Psychology, 2, 391-410. doi: 10.1007/s13164016-0308-1

Leichsenring, F., Luyten, P., Hilsenroth, M. J., Abbass, A., Barber, J. P., Keefe, J. R., ... Steinert, C. (2015). Psychodynamic therapy meets evidence-based medicine: a systematic review using updated criteria. The Lancet Psychiatry, 2, 648-660. doi/10.1016/S2215-0366(15)00155-8

Mansell, W. (2007). An integrative formulation-based cognitive treatment of bipolar disorders: application and illustration. Journal of Clinical Psychology, 63, 447-461

Mansell, W., Carey, T. A., \& Tai, S. J. (2013). A transdiagnostic approach to CBT using Method of Levels Therapy. East Sussex: Routledge.

Nathan, P. E. \& Gorman, J. M. (2015). A guide to treatments that work (4th. ed.). Oxford: Oxford University Press.

Osbeck, L. M. (2009). Transformations in Cognitive Science: Implications and Issues. Journal of Theoretical and Philosophical Psychology, 29, 16-33. DOI: 10.1037/a0015454
Paris, J. (2013). How the history of psychotherapy interferes with integration. Journal of Psychotherapy Integration, 23, 99106.

Rabinovich, M. (2016). Psychodynamic emotional regulation in view of Wolpe's desensitization model. American Journal of Psychology, 129, 65-79. dOI: 10.5406/amerjpsyc.129.1.0065

Rosner, R., Bartl, H., Pfoh, G., Kotoucova, M., \& Hagl, M. (2015). Efficacy of an integrative CBT for prolonged grief disorder: a long-term follow up. Journal of Affective Disorders, 183, 106-12. doi: 10.1016/j.jad 201504.051

Sánchez-Arribas, C., Chorot, P., Valiente, R. M., y Sandín, B. (2015). Evaluación de factores cognitivos positivos y negativos relacionadas con el trastorno de pánico: Validación del CATP. Revista de Psicopatología y Psicología Clínica, 20, 85-100. doi: 10.5944/rppc.vol.20.num.2.2015.15164

Sandin, B., Sánchez-Arribas, C., Chorot, P., \& Valiente, R. M. (2015). Anxiety sensitivity, catastrophic misinterpretations and panic self-efficacy in the prediction of panic disorder severity: towards a tripartite cognitive model of panic disorder. Behaviour Research and Therapy, 67, 30-40. doi: 10.1016/j. brat 201501.005

Swenson, C. R. \& Choi-Kain, L. W. (2015). Mentalization and Dialectical Behavior Therapy. American Journal of Psychotherapy, 69, 199-217.

Wampold, B. (2015). How important are the common factors in psychotherapy? An update. World Psychiatry, 14, 270-277.

Wilkowski. B. M. \& Robinson, M. D. (2010). The anatomy of anger: an integrative cognitive model of trait anger and reactive aggression. Journal of Personality, 78, 9-38. doi: 10.1111/j.1467-6494.2009.00607

Wonderlich, S. A., Peterson, C. B., \& Smith, T. L. (2015). Integrative cognitive-affective therapy for bulimia nervosa: A treatment manual. New York: Guilford

Young, J. E., Klosko, J. S., \& Weishaar, M. E. (2003). Schema Therapy: A practitioner's guide. New York: Guilford. 
